\title{
Effect of bronchodilation on expiratory flow limitation and resting lung mechanics
} in COPD

\author{
R.L. Dellacà*, P.P. Pompilio*, P.P. Walker*, N. Duffy", \\ A. Pedotti* and P.M.A. Calverley ${ }^{\#}$
}

ABSTRACT: Bronchodilator drugs produce variable improvements in forced expiratory volume in $1 \mathrm{~s}$ (FEV 1), but larger changes in end-expiratory lung volume (EELV) in chronic obstructive pulmonary disease (COPD), which were suggested to be related to the presence of expiratory flow limitation (EFL) at rest.

We tested this concept in 42 COPD patients (FEV $142.3 \pm 13.8 \%$ predicted) during spontaneous breathing before and after $5 \mathrm{mg}$ nebulised salbutamol. EFL was detected by within-breath changes in respiratory system reactance measured by a multifrequency forced oscillation method, while changes in EELV were assessed by inspiratory capacity (IC). Bronchodilation (BD) increased IC (from $1.8 \pm 0.5$ to $2.1 \pm 0.6 \mathrm{~L}, \mathrm{p}<0.001$ ) and reduced inspiration resistance $(\bar{R}$ insp) at $5 \mathrm{~Hz}$ (from $5.1 \pm 1.6$ to $4.2 \pm 1.5 \mathrm{cmH}_{2} \mathrm{O} \cdot \mathrm{s} \cdot \mathrm{L}^{-1}, \mathrm{p}<0.001$ ). $\bar{R}$ insp identified BD responders with a discriminative power of $80.1 \%$.

In total, 20 patients were flow-limited before BD. They showed worse spirometry and higher residual volume, but significant improvements in IC were seen in all patients irrespective of flow limitation. Changes in $\overline{\boldsymbol{R}}$ insp were confined to flow-limited patients, as were reactance changes. BD reduced the degree of heterogeneity in the respiratory system, a change best seen with inspiratory values.

BD has complex effects on lung mechanics in COPD, and EFL affects both this and the response of some respiratory variables to treatment. However, changes in EELV are consistently seen, irrespective of the presence of flow limitation at rest.

KEYWORDS: Chronic obstructive pulmonary disease, forced oscillation technique, respiratory system reactance, within-breath impedance

hronic obstructive pulmonary disease (COPD) is defined by the presence of incompletely reversible expiratory airflow limitation (EFL) [1], which occurs at much lower flows for any given lung volume when compared with healthy subjects. Initially, flow limitation is only present during maximal or near maximal respiratory efforts, but as lung disease progresses, EFL develops at rest in many, but not all, individuals [2]. The presence of resting EFL may identify COPD patients who behave differently and who develop dynamic hyperinflation [3], at least during exercise [4].

Bronchodilator drugs improve lung emptying, and this leads to variable increases in forced expiratory volume in $1 \mathrm{~s}$ (FEV1), mainly by reducing lung volume rather than changing the FEV1/forced vital capacity (FVC) ratio [5]. However, the reproducibility and predictive value of testing for FEV1 reversibility is relatively poor $[6,7]$, while the change in resting inspiratory capacity has been shown to be a better predictor of improvement in exercise performance $[8,9]$. Again, patients with EFL have been reported to show improvements in inspiratory capacity after bronchodilators [10], which may relate to an improvement in exercise performance [11].

Previous workers have used the negative expiratory pressure method to detect EFL [12], but that study samples a relatively small number of breaths and not all tests are suitable for analysis [2]. We
AFFILIATIONS

*TBM Lab, Dipartimento di Bioingegneria, Politecnico di Milano University, Milano, Italy. \#University Hospital Aintree, University Dept of Medicine, Liverpool, UK.

CORRESPONDENCE

R.L. Dellacà

Dipartimento di Bioingegneria

Politecnico di Milano

Piazza Leonardo da Vinci 32

I-20133 Milano

Italy

Fax: 390223999000

E-mail: raffaele.dellaca@polimi.it

Received:

September 102008

Accepted after revision:

December 292008

\section{SUPPORT STATEMENT}

The present work was partially supported by the British Lung Foundation. R.L. Dellacà has recieved a European Respiratory Society Fellowship (no. 43)

\section{STATEMENT OF INTEREST} Statements of interest for R.L. Dellacà, P.P. Pompilio and A. Pedotti can be found at

www.erj.ersjournals.com/misc/ statements.dtl 
have developed an effort-independent method to determine flow limitation during tidal breathing using the forced oscillatory technique to identify within-breath differences in respiratory system reactance [13, 14]. This method allows the assessment of more breaths, is equivalent to the negative expiratory pressure approach when both can be recorded [2], and adds a potential "quantitative" assessment of how close the patent is to the threshold of EFL [14]. Modelling simulations based on these data suggest that EFL will influence other measurements of oscillatory mechanics during expiration, and this will reduce the sensitivity of expiratory impedance data to change after interventions, such as bronchodilators. Although this effect can be identified when within-breath analysis is performed [13], most published reports of oscillatory mechanics in COPD only report total respiratory cycle data [15-17].

In the current study, we tested the hypothesis that the changes in lung volume (specifically inspiratory capacity) and oscillatory lung mechanics of COPD patients given an inhaled bronchodilator drug would differ when EFL was present, and whether this would be unrelated to the presence of reversibility defined spirometrically. Additionally, we extended our observations of within-breath impedance using a forced oscillation method from single to multiple forcing frequencies. This approach allowed us to test whether bronchodilator drugs improve resistance and the intrapulmonary homogeneity of lung mechanics, avoiding the confounding effects of EFL on impedance data that would be corrupted when adopting the conventional multifrequency approach. Finally, we examined the changes in resting lung and respiratory system mechanics in those individuals who were no longer flow-limited after the bronchodilator drug.

\section{METHODS \\ Subjects}

We recruited clinically stable outpatients who met the diagnostic criteria for COPD [18] and were either current or ex-smokers. All patients were using short- and long-acting inhaled bronchodilators, which were omitted before study for 3-24 h, as appropriate. The study was approved by the institutional research ethical review committee (South Sefton Research Ethics Committee, Liverpool, UK), and written informed consent was given by each subject.

\section{Measurements}

Forced expiratory flow, lung volume and subdivisions were measured by a constant-volume body plethysmograph (Medgraphic Autolink 1085D; Medical Graphics, St Paul, MN, USA). All measurements met current standards for acceptable data quality [19]. We report FEV1, FVC, FEV1/FVC, inspiratory capacity (IC), residual volume (RV), thoracic gas volume (TGV) and total lung capacity (TLC) both as absolute values and \% predicted ( $\%$ pred). Predicted values were those recommended by the European Respiratory Society (ERS) [20].

We measured breathing pattern and oscillatory mechanics using previously described methods [13]. Briefly, we recorded pressure and flow at the airway opening by a transducer connected to the mouthpiece (PXLA0025DN; Sensym, Milpitas, CA, USA) and by a screen-type pneumotachograph (3700A, Hans Rudolph, Kansas City, MO, USA) connected to another pressure transducer (PXLA02X5DN, 0-2.5 cm; Sensym). All the signals were sampled at $200 \mathrm{~Hz}$ and recorded onto a PC. The flow signal was integrated to give lung volume, and volume drift was removed by selecting 2-3 min of stable quiet breathing and estimating the linear trend on the integrated signal. This trend was then removed from the traces.

From these signals we measured tidal volume $(V T)$, respiratory frequency, total cycle duration, inspiratory time, expiratory time and inspiratory duty cycle (fig. 1). We derived minute ventilation $\left(V^{\prime} \mathrm{E}\right)$, mean inspiratory flow rate and mean expiratory flow rate from these data.

\section{Forced oscillations}

Patients were studied while being oscillated by the following two different waveforms: 1) a $5 \mathrm{~Hz}$ sinusoidal signal, and 2) a pseudo-random noise (PRN) with three components at 5, 11 and $19 \mathrm{~Hz}$ chosen to be non-sum non-difference of order 3 [21]. For both the waveforms, the peak-to-peak pressure amplitude measured at the mouth was $\sim 1-2 \mathrm{cmH}_{2} \mathrm{O}$. In order to have comparable total energy at $5 \mathrm{~Hz}$ in both the sinusoidal and the

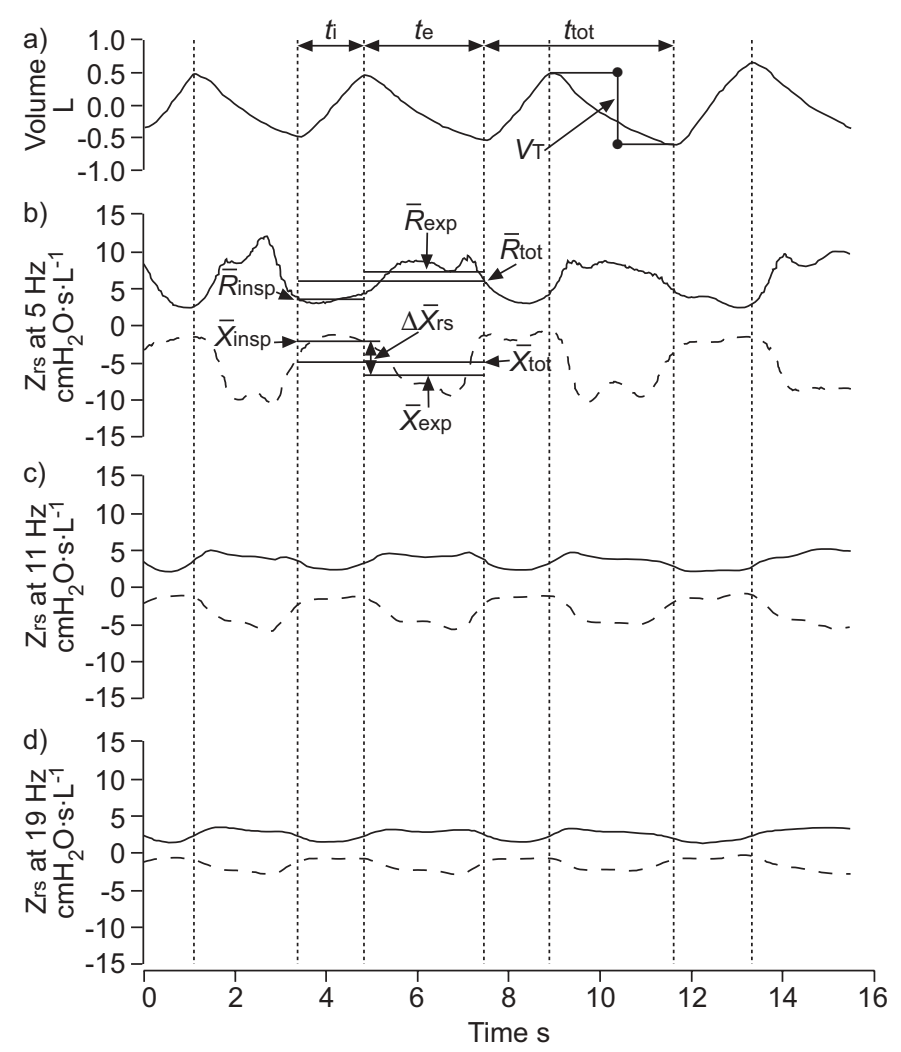

FIGURE 1. Examples of a representative experimental tracing of volume and within-breath multifrequency impedance from a chronic obstructive pulmonary disease patient with the definition of the indices considered in our study. a) Volume measured by integration of flow at the mouth. b)-d) Within-breath respiratory impedance $(\mathrm{Zrs})$ at $5 \mathrm{~Hz}(\mathrm{~b}), 11 \mathrm{~Hz}(\mathrm{c})$ and $19 \mathrm{~Hz}(\mathrm{~d})$. Each impedance is expressed as respiratory system resistance ( $R r s,-\longrightarrow)$ and reactance ( $\left.X_{r s},------\right)$. The presence of large within-breath variation of $X_{r s}$ with more negative values showed during expiration is a clear sign of the presence of expiratory flow limitation. $t$ i: inspiratory time; te: expiratory time; ttot: total cycle duration; VT: tidal volume; $\bar{R} \exp$ : mean expiratory resistance; $\bar{R}$ tot: mean whole-breath resistance; $\bar{R}$ insp: mean inspiratory resistance; $\bar{X}_{\text {insp: }}$ mean inspiratory reactance; $\Delta \bar{X}_{\text {rs: }}$ difference between $\bar{X}_{\text {insp }}$ and $\bar{X}_{\text {esp. }} . \bar{X}_{\text {tot: }}$ mean whole breath reactance; $\bar{X}_{\text {exp: }}$ mean expiratory reactance. 
PRN signals, and to keep the total energy of the PRN signal low, the relative amplitude of the $5 \mathrm{~Hz}$ component of the PRN has been slightly increased.

The experimental set-up for forced oscillation technique (FOT) measurement was similar to that described previously [13]. The pressure signal generated by a loudspeaker was transferred from the box to the subject's mouthpiece through a connecting tube (22 cm long, $19 \mathrm{~mm}$ internal diameter). A low-resistance, highly inert tube (1.5 m long, $22 \mathrm{~mm}$ internal diameter) in parallel with the loudspeaker allowed the subjects to breathe room air without significant loss of forcing pressure. A bias flow of $\sim 15 \mathrm{~L} \cdot \mathrm{min}^{-1}$ reduced the equipment dead space to the volume of the pneumotachograph and the mouthpiece [22]. The frequency response of the whole measuring system was assessed up to $30 \mathrm{~Hz}$, as described previously [2], and was flat.

\section{Experimental protocol}

Patients attended on one occasion, when all measurements were made in the same order. Plethysmographic lung volume measurements were followed by recording oscillatory mechanics at $5 \mathrm{~Hz}$ and breathing pattern with the patients seated, wearing a nose-clip and with an operator firmly supporting the cheeks to reduce upper airways shunt [23]. The patients breathed spontaneously through the FOT system for $1 \mathrm{~min}$, then performed an IC manoeuvre and resumed spontaneous breathing.

After 10-min rest with the patient disconnected from the measuring circuit, the FOT measurements were repeated by following the same sequence of manoeuvres, but with the multifrequency PRN signal applied.

Next, patients received $5 \mathrm{mg}$ of nebulised salbutamol from an oxygen-driven Acorn ${ }$ nebuliser (MedicAid, Pagham, UK) and after $45 \mathrm{~min}$ of spirometry, plethysmography and the two FOT measurements were repeated as described previously.

\section{Data analysis}

Within-breath respiratory system input impedance (Zrs) was determined by using a least squares algorithm taking advantage of the a priori knowledge of the frequency spectrum components of the forcing signals [24, 25].

From the complete FOT recording and impedance tracing, we selected $\sim 10$ breaths starting from $45 \mathrm{~s}$ after the first IC, to avoid possible alterations of the breathing pattern after the manoeuvre. Breaths in which Zrs tracings showed spikes or oscillations due to swallowing or glottis closure were discarded. For each breath, the values of several breathing pattern parameters and $\mathrm{Zrs}$ indices were computed and averaged for all the breaths in each subject and condition. Within-breath respiratory system resistance (Rrs) was characterised by the mean values it assumed during inspiration, expiration and during the whole breath ( $\bar{R}_{\text {insp, }} \bar{R} \exp$ and $\bar{R}$ tot, respectively). As the frequency dependence of $R$ rs is related to the heterogeneity of airway obstruction [22, 26], we also computed the difference between $R$ rs measured at our lowest and highest frequency ( 5 and $19 \mathrm{~Hz}, \bar{R} 5-\bar{R} 19)$.

Respiratory system reactance (Xrs) was characterised by its average value during a breath and its within-breath fluctuations were quantified by computing its average value during inspiration $\left(\bar{X}_{\text {insp }}\right)$ and expiration $\left(\bar{X}_{\text {exp }}\right)$. Their difference $\left(\Delta \bar{X}_{\mathrm{rs}}=\bar{X}_{\text {insp- }} \bar{X}_{\text {exp }}\right)$ was used to detect EFL. A breath was considered flow-limited if $\Delta \bar{X}$ rs was greater than a threshold of $2.8 \mathrm{cmH}_{2} \mathrm{O} \cdot \mathrm{s} \cdot \mathrm{L}^{-1}$, a value that in our previous studies $[2,13$, $14]$, enabled identification of flow-limited breaths with very high sensitivity and specificity. A subject was classified as flow-limited if the majority of his selected breaths were flowlimited.

Data are expressed as mean $\pm \mathrm{SD}$, unless otherwise stated. All data comparisons were made relative to that individual's baseline value, although we did conduct an exploratory analysis of the spirometry data based on the reversibility criteria recommended by the American Thoracic Society (ATS)/ERS to identify bronchodilator responsiveness (FEV1 change $>12 \%$ from the baseline and $>200 \mathrm{~mL}$ ) [27]. Significant differences in the physical characteristics, spirometric data, and $R$ rs and $X$ rs indices values of the different groups were evaluated using paired or unpaired t-tests, as appropriate. To allow for the multiple comparisons to be made between groups, only $\mathrm{p}<0.01$ was considered to be statistically significant. Our primary outcome was the change in IC after administration of the bronchodilator. We calculated that a study with 15 patients would have an $80 \%$ chance of showing a difference of $200 \mathrm{~mL}$ at the $5 \%$ significance level between the groups. As we anticipated identifying flow-limited and nonflow-limited patients, we aimed to recruit 40 individuals to increase our ability to detect differences between the subgroups.

\section{RESULTS}

The baseline characteristics of the 42 COPD patients recruited in this study are reported in table 1 . All patients performed the measurements correctly, with no reports of discomfort. From these patients, a total of 788 breaths were selected and analysed (408 before and 380 after bronchodilator). In figure 1, an experimental tracing of volume and multifrequency impedance data are shown for a representative flow-limited patient. The presence of flow limitation is clearly shown by the large decrease of $X \mathrm{rs}$ during expiration compared with inspiration. Figure 1 also shows that the presence of EFL affects within-breath variations of $Z$ rs at all frequencies but, as predicted by the model simulation [13], the intra-breath Xrs swings decrease in amplitude with increasing frequencies.

The $5 \mathrm{~Hz}$ component of the multifrequency forcing gave similar results to those of the single $5 \mathrm{~Hz}$ frequency and, therefore, in the rest of this study only data recorded during multifrequency forcing are reported. A full account of the comparison of the single and multifrequency testing is presented in the online supplementary material.

\section{Group mean data post-bronchodilator without accounting for tidal expiratory flow limitation}

The lung function, breathing pattern and impedance indices for the whole patient group, measured after the bronchodilator are presented in tables 1 and 2. Bronchodilation (BD) produced statistically significant improvements in all the measured plethysmographic variables except for FEV1/FVC and TLC (table 1). There was a significant increase in $V^{\prime} \mathrm{E}$ and a fall in mean inspiratory and expiratory flow (table 2). 


\begin{tabular}{|c|c|c|c|}
\hline \multirow[t]{2}{*}{ TABLE 1} & \multicolumn{3}{|c|}{$\begin{array}{l}\text { Patient characteristics and lung function before } \\
\text { and after bronchodilation (BD) }\end{array}$} \\
\hline & Pre-BD & Post-BD & $p$-value \\
\hline Age yrs & $63.7 \pm 8.4$ & & \\
\hline Sex $M / F$ & $21 / 23$ & & \\
\hline Weight kg & $68.3 \pm 21.6$ & & \\
\hline Height $\mathrm{cm}$ & $163.9 \pm 9.0$ & & \\
\hline \multicolumn{4}{|l|}{ FEV $_{1}$} \\
\hline L & $1.12 \pm 0.39$ & $1.31 \pm 0.46$ & $<0.001$ \\
\hline$\%$ pred & $42.28 \pm 13.82$ & $49.08 \pm 15.87$ & $<0.001$ \\
\hline FEV $_{1 / F V C}$ & $46.81 \pm 10.75$ & $46.23 \pm 11.59$ & 0.510 \\
\hline \multicolumn{4}{|l|}{ FVC } \\
\hline L & $2.42 \pm 0.68$ & $2.86 \pm 0.76$ & $<0.001$ \\
\hline$\%$ pred & $68.69 \pm 14.26$ & $80.99 \pm 15.14$ & $<0.001$ \\
\hline \multicolumn{4}{|l|}{ svc } \\
\hline $\mathrm{L}$ & $2.60 \pm 0.70$ & $2.95 \pm 0.83$ & $<0.001$ \\
\hline$\%$ pred & $73.01 \pm 12.64$ & $82.78 \pm 16.33$ & $<0.001$ \\
\hline \multicolumn{4}{|l|}{ IC } \\
\hline L & $1.85 \pm 0.52$ & $2.09 \pm 0.62$ & $<0.001$ \\
\hline$\%$ pred & $72.95 \pm 17.94$ & $81.51 \pm 18.92$ & $<0.001$ \\
\hline \multicolumn{4}{|l|}{ RV } \\
\hline L & $4.82 \pm 1.31$ & $4.50 \pm 1.15$ & $<0.001$ \\
\hline$\%$ pred & $235.00 \pm 59.04$ & $219.99 \pm 50.84$ & $<0.001$ \\
\hline \multicolumn{4}{|l|}{ TGV } \\
\hline L & $5.62 \pm 1.48$ & $5.35 \pm 1.37$ & $<0.001$ \\
\hline$\%$ pred & $184.49 \pm 36.19$ & $175.60 \pm 34.23$ & $<0.001$ \\
\hline \multicolumn{4}{|l|}{ TLC } \\
\hline $\mathrm{L}$ & $7.50 \pm 1.72$ & $7.44 \pm 1.65$ & 0.228 \\
\hline$\%$ pred & $131.74 \pm 20.03$ & $130.86 \pm 17.73$ & 0.175 \\
\hline
\end{tabular}

Data are presented as mean $\pm \mathrm{SD}$, unless otherwise indicated. $\mathrm{M} / \mathrm{F}$ : male/ female; FEV1: forced expiratory volume in $1 \mathrm{~s}$; \% pred: \% predicted; FVC: forced vital capacity; SVC: slow vital capacity; IC: inspiratory capacity; RV: residual volume; TGV: thoracic gas volume; TLC: total lung capacity.

In total, 18 patients met the ATS/ERS criteria for reversibility of airway obstruction. There were no differences between responder and nonresponder groups in their baseline plethysmographic or oscillatory variables. The changes in plethysmographic variables post-bronchodilator were similar for the two spirometrically defined groups, while the oscillometric indices differed between the responders and nonresponders. Specifically $\bar{R}$ tot and $\bar{R}$ insp at $5 \mathrm{~Hz}$ and mean difference in resistances at 5 and $19 \mathrm{~Hz}(\bar{R} 5-\bar{R} 19)$ fell significantly more $(\mathrm{p}=0.002, \mathrm{p}=0.001$ and $\mathrm{p}=0.002$, respectively), while no differences were seen in $X r$ between the groups. The discriminative power tested by the receiver operated characteristic curves was greater when $\bar{R}$ insp was used compared with $\bar{R}$ tot $(80.1 \%$ and $73.5 \%$, respectively) [28]. More details on lung volumes, breathing pattern and Zrs data for responders and nonresponders groups are shown in table E4 in the online supplementary material.

In general, changes in Xrs indices were statistically significant at all forcing frequencies, while the fall in Rrs only occurred consistently when measured during inspiration. As a result $\bar{R}$ tot only decreased significantly relative to baseline at $5 \mathrm{~Hz}$ while $\bar{R} \exp$ did not change significantly at any frequency.

\begin{tabular}{|c|c|c|c|}
\hline \multirow[t]{2}{*}{ TABLE 2} & \multicolumn{3}{|c|}{$\begin{array}{l}\text { Breathing pattern and within-breath input } \\
\text { impedance measured at } 5 \mathrm{~Hz} \text { data for patients } \\
\text { before and after bronchodilation (BD) }\end{array}$} \\
\hline & Pre-BD & Post-BD & $p$-value \\
\hline$V^{\prime} E L \cdot \min ^{-1}$ & $11.036 \pm 3.465$ & $12.053 \pm 3.731$ & $<0.001$ \\
\hline VT L & $0.656 \pm 0.240$ & $0.711 \pm 0.244$ & 0.014 \\
\hline$f_{R}$ breaths $\min ^{-1}$ & $18.1 \pm 5.6$ & $18.1 \pm 5.4$ & 0.936 \\
\hline tis & $1.375 \pm 0.473$ & $1.373 \pm 0.540$ & 0.975 \\
\hline tot $\mathrm{S}$ & $3.747 \pm 1.525$ & $3.743 \pm 1.633$ & 0.968 \\
\hline$t_{\mathrm{i}} /$ ttot & $0.376 \pm 0.057$ & $0.375 \pm 0.055$ & 0.723 \\
\hline$V T / t i \mathbf{L} \cdot \mathbf{s}^{-1}$ & $0.500 \pm 0.169$ & $0.545 \pm 0.167$ & $<0.001$ \\
\hline$V T / t E L \cdot S^{-1}$ & $0.299 \pm 0.095$ & $0.327 \pm 0.108$ & 0.001 \\
\hline $\bar{R}$ insp & $5.1 \pm 1.6$ & $4.2 \pm 1.5$ & $<0.001$ \\
\hline $\bar{R} \exp$ & $6.2 \pm 2.5$ & $5.8 \pm 2.7$ & 0.036 \\
\hline $\bar{R}$ tot & $5.8 \pm 2.1$ & $5.2 \pm 2.2$ & 0.001 \\
\hline $\bar{X}_{\text {insp }}$ & $-2.3 \pm 1.2$ & $-1.8 \pm 1.1$ & $<0.001$ \\
\hline $\bar{X}_{\exp }$ & $-5.3 \pm 4.3$ & $-3.8 \pm 3.3$ & 0.001 \\
\hline $\bar{X}_{\text {tot }}$ & $-4.3 \pm 3.3$ & $-3.1 \pm 2.5$ & 0.001 \\
\hline$\Delta \overline{\mathbf{X}}_{\mathrm{rs}}$ & $2.9 \pm 3.4$ & $2.0 \pm 2.5$ & 0.004 \\
\hline $\bar{R} 5-\bar{R} 19$ & $1.8 \pm 1.0$ & $1.2 \pm 0.9$ & $<0.001$ \\
\hline
\end{tabular}

Data are presented as mean $\pm \mathrm{SD}$, unless otherwise indicated. Impedance data $(R$ and $X)$ are expressed as $\mathrm{cmH}_{2} \mathrm{O} \cdot \mathrm{s} \cdot \mathrm{L}^{-1} \cdot V^{\prime} \mathrm{E}$ : minute ventilation; $V \mathrm{~T}$ : tidal volume; fR: respiratory frequency; ti: inspiratory time; ttot: total cycle duration; ti/tot: inspiratory duty cycle; $V_{T} / t i$ : mean respiratory flow rate; $V_{T} / t \mathrm{E}$ : mean expiratory flow rate; $\bar{R}$ insp: mean inspiration resistance; $\bar{R}$ exp: mean expiration resistance; $\bar{R}$ tot: mean whole breath resistance; $\bar{X}_{\text {insp: }}$ mean reactance during inspiration; $\bar{X}_{\text {exp }}$; mean reactance during expiration: $\bar{X}_{\text {tot: }}$ mean total reactance ; $\Delta \bar{X}_{\text {rs: }}$ mean difference in reactance; $\bar{R} 5-\bar{R} 19$ : mean difference in resistances at 5 and $19 \mathrm{~Hz}$.

Considering all the patients, $\bar{R}$ insp at $5 \mathrm{~Hz}$ was statistically greater than at $19 \mathrm{~Hz}$ both before and after BD. However, it was possible to identify a subgroup of seven patients in which this difference was statistically different before BD $(p=0.008)$, but not after $(p=0.204)$ BD. These patients were, on average, less obstructed (FEV1 was $56.6 \pm 16.02 \%$ predicted (\% pred) pre- and $66.9 \pm 18.6 \%$ pred post-BD) than the others, enforcing the concept that $\bar{R} 5-\bar{R} 19$ can be used as a sensitive index of heterogeneity in airway obstruction. Indeed, $\bar{R} 5-\bar{R} 19$ showed a statistically significant decrease, suggesting that the pattern of airway obstruction was on average more homogeneous after BD (table 2, fig. 2).

\section{Effects of expiratory flow limitation on pre- and post- bronchodilator lung function and impedance measurements}

Of the 42 patients, 20 were flow-limited at rest pre-bronchodilator and EFL was present in the majority of breaths studied both before and after BD (fig. 3).

At baseline, FEV1 was clearly lower and RV higher in the flowlimited patients, but the differences in other plethysmographic variables did not reach our adjusted significance level.

Of the oscillometric measurements $\bar{R}$ insp, $\bar{R} 5-\bar{R} 19$ and all the reactance indices were greater in the flow-limited patients, 

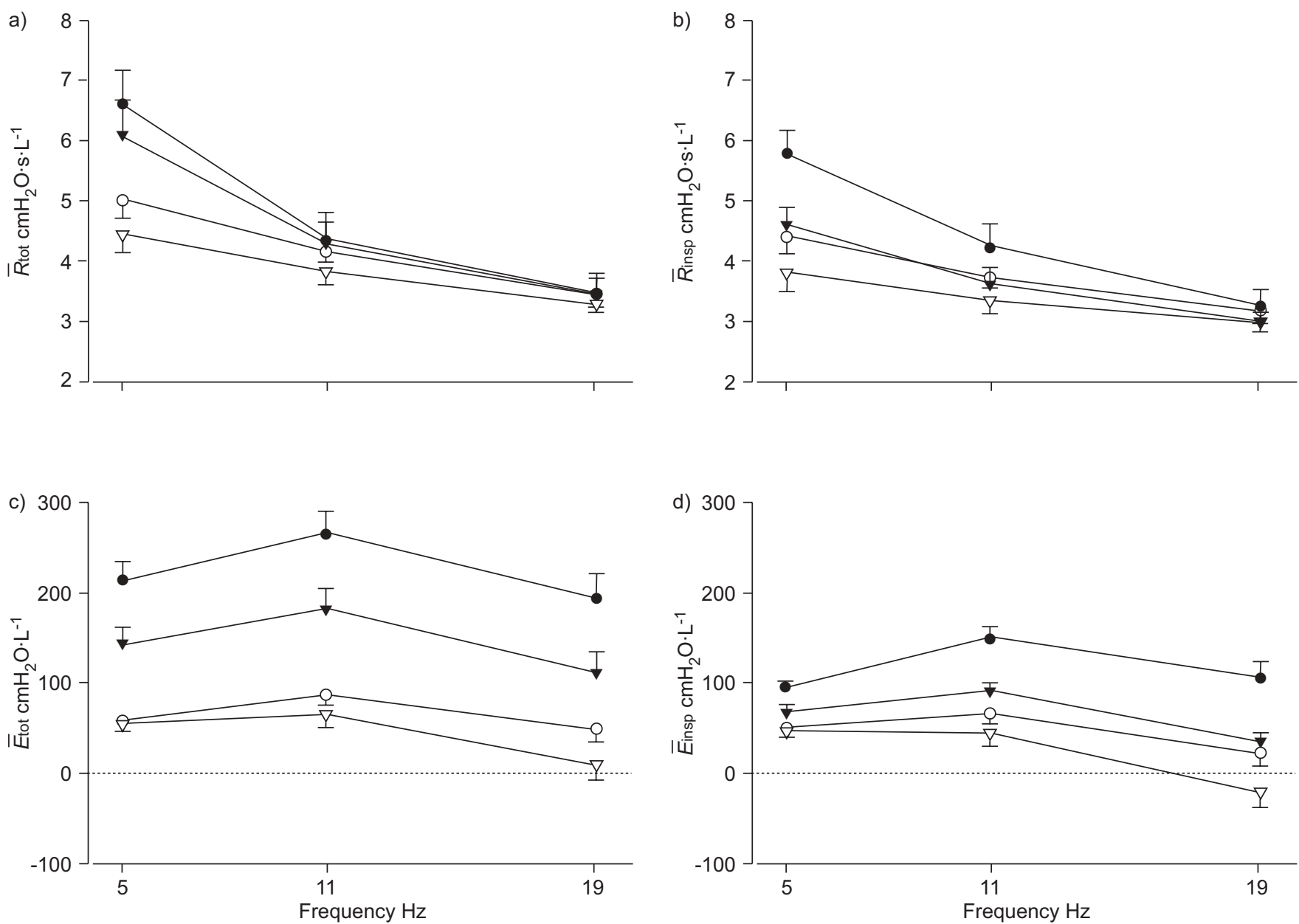

FIGURE 2. Multifrequency impedance spectra for a) mean whole breath resistance ( $\bar{R}$ tot), b) mean inspiration resistance ( $\bar{R}$ insp), c) mean whole breath respiratory elastance at $5 \mathrm{~Hz}$ (E $\left.\bar{E}_{\text {tot }}\right)$ and d) mean inspiratory elastance at $5 \mathrm{~Hz}$ (Ëinsp) in flow-limited (closed symbols) and nonflow-limited (open symbols) patients at baseline before (circles) and after (triangles) bronchodilation.

with nonsignificant differences being seen in the other resistance measurements.

Post-bronchodilator, both groups improved in all spirometric and lung volume variables except for FEV1/FVC and TLC (table 3, fig. 4a). There were significant decreases in $\bar{R}$ insp and $\bar{R}$ tot in patients with EFL, but not in those without EFL where the pre-bronchodilator values for these variables were significantly lower (table 3, fig. 4b). Xrs was significantly less negative after $\mathrm{BD}$ at all frequencies in flow-limited patients, while in nonflow-limited subjects, the change in Xrs was significant only at high frequencies.

\section{The effect of flow limitation on indices of lung homogeneity pre- and post-BD}

To further investigate the effect of the bronchodilator without the confounding effect of expiratory flow limitation, we considered both $\bar{R}$ tot and $\bar{R}$ insp, and dynamic elastance (i.e. $X$ rs multiplied by $-2 \pi f$, where $f$ is the forcing frequency) over the forcing frequencies used in this study (fig. 2). Unlike patients without flow limitation, EFL patients showed a clear pattern of frequency dependence that became more evident when the expiratory phase was excluded. After the bronchodilator, Rrs decreased at all frequencies in nonflow-limited patients, and the changes were similar whether $\bar{R}$ tot or $\bar{R}$ insp data were selected. By contrast, the change in Rrs was much more evident (and statistically significant) in EFL patients when $\bar{R}$ insp was used (fig. 2).

\section{Effects of a bronchodilator on the presence of expiratory flow limitation}

Of the 20 flow-limited patients at baseline, eight patients became nonflow-limited after BD, while no patient initially without EFL developed it. The patients where flow limitation was abolished had nonsignificantly different $\Delta \bar{X}$ rs values at baseline and changes in the $\Delta \bar{X}$ rs after salbutamol compared with those patients where flow-limitation persisted. There was no relationship between inspiratory capacity and $\Delta \bar{X}$ rs changes overall in the flow-limited patients $(\mathrm{r}=0.107)$.

\section{DISCUSSION}

The development of expiratory flow limitation during tidal breathing identifies a group of COPD patients whose ability to increase their $V \mathrm{~T}$ to maintain gas exchange is significantly 


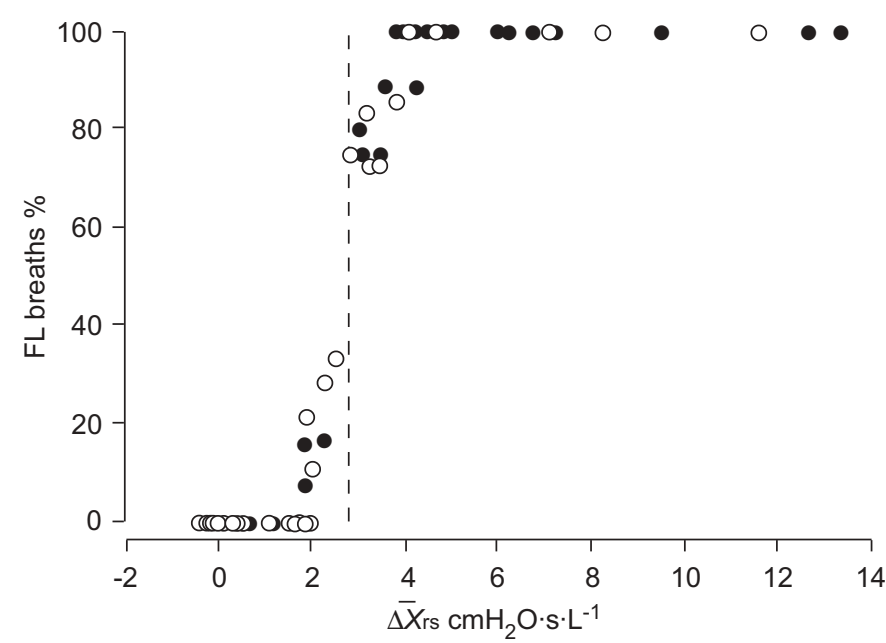

FIGURE 3. Relationship between the average value of mean difference in respiratory system reactance $\left(\Delta \bar{X}_{\mathrm{rs}}\right)$ and the percentage of individually classified flow-limited $(\mathrm{FL})$ breath for each patient before $(\bullet)$ and after $(O)$ bronchodilation.

limited [29]. Inhaling a bronchodilator drug can potentially have multiple effects in COPD, which may be influenced by the presence of tidal EFL. These include a reduction in airways' resistance, an abolition of EFL at that operating lung volume or a shift in the distribution of choke points within the lung, all of which can lead to a fall in end-expiratory lung volume that in turn may lead to the persistence of EFL at rest. We used the forced oscillation method to identify the presence of expiratory flow limitation on a breath-by-breath basis, to measure respiratory system mechanics during tidal breathing and to quantify the heterogeneity of lung obstruction in COPD. To do this we used a within-breath multifrequency method that allowed us to assess the heterogeneity of the obstruction [26, 30], and produced comparable data to that measured using the single frequency approach. Our data in a more homogeneous patient group suggest that the response to bronchodilators is more complex than initially proposed [10].

The effect of the high dose $\beta$-agonist on resting lung mechanics and breathing pattern we observed in the group as a whole was similar to that reported in other studies of hyperinflated COPD patients $[5,9,31]$, with significant increases in FEV1 and inspiratory capacity and falls in RV and TGV. $V^{\prime}$ E increased, as did mean inspiratory and expiratory flow rates, compatible with the decrease in total and inspiratory resistance. Bronchodilator reversibility defined spirometrically is common in COPD [32]. Although nearly half of our patients met the current criteria for a response [27], there was no difference in the magnitude of the IC change in spirometric responders and nonresponders, which helps to explain why these tests are only poorly predictive of the patient's subsequent clinical course $[6,7,33]$. However, oscillatory mechanics changed differently in responders and nonresponders, with the Rrs values tracking the changes in FEV1, unlike the reactance values, which followed the inspiratory capacity data. A similar discrepancy between resistance and reactance measurements pattern has been reported during recovery from COPD exacerbations $[34,35]$, where changes in breathlessness follow those in inspiratory capacity.
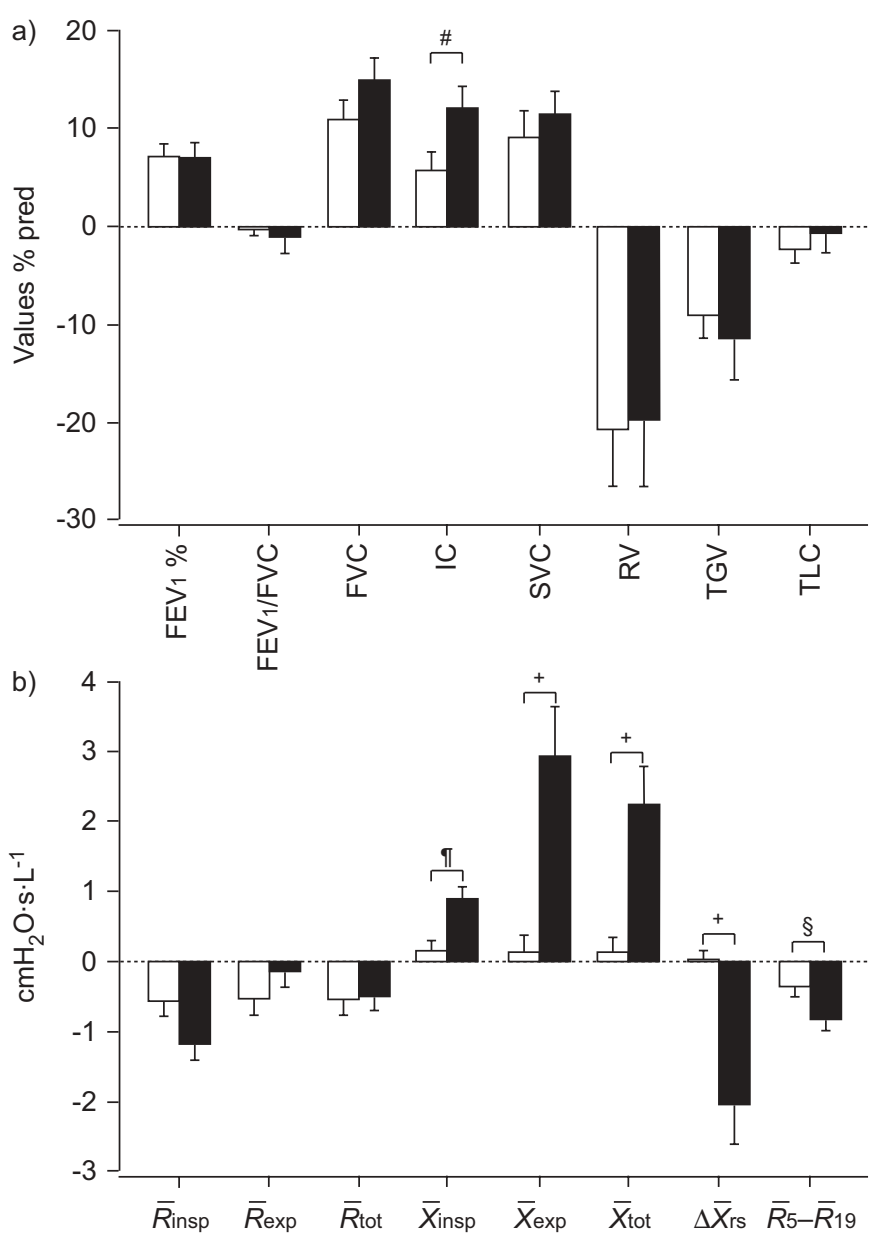

FIGURE 4. Changes of a) spirometric, plethysmographic, and b) impedance data at $5 \mathrm{~Hz}$ induced by bronchodilator in flow-limited $(\square)$ and nonflow-limited (ם) patients at baseline. \% pred: \% predicted; FEV1: forced expiratory volume in $1 \mathrm{~s}$; FVC: forced vital capacity; IC: inspiratory capacity; SVC: slow vital capacity; RV: residual volume; TGV: thoracic gas volume; TLC: total lung capacity; $\bar{R}$ insp: mean inspiration resistance; $\bar{R}$ exp: mean expiration resistance; $\bar{R}$ tot: mean whole breath resistance; $\bar{X}_{\text {insp: }}$ mean reactance during inspiration; $\bar{X}_{\text {exp }}$; mean reactance during expiration: $\bar{X}_{\text {tot: }}$ mean total reactance ; $\Delta \bar{X}_{\text {rs: }}$ mean difference in reactance; $\bar{R} 5-\bar{R}$ 19: mean difference in resistances at 5 and $19 \mathrm{~Hz}$. ${ }^{*}: \mathrm{p}=0.04 ;{ }^{\circ}: \mathrm{p}=0.05$; $+: p=0.001 ;$ s: $p=0.031$.

Resting expiratory flow limitation was present in just over half the patients. All the breaths studied were consistently classified, except for eight cases where the degree of EFL varied from breath to breath and the classification was based on a majority decision. As expected, flow-limited patients had worse spirometry and a higher RV with a general tendency for higher lung volumes, although these differences were less consistent between groups. Despite this, the response to bronchodilators was almost identical with similar changes in flow and volume indices irrespective of the presence of flow limitation. This does not preclude a different behaviour during exercise in the patients who were flow-limited at rest, but the improvement in exercise performance post-bronchodilator has been consistently related to changes in resting inspiratory capacity, without reference to whether these occurred in flowlimited patients $[33,36]$. 
TABLE 3 Lung function, breathing pattern and impedance data at $5 \mathrm{~Hz}$ for patients who were nonflow-limited (non-FL) at baseline and patients who were FL

\begin{tabular}{|c|c|c|c|c|c|c|c|}
\hline & \multicolumn{2}{|c|}{ Non-FL at baseline } & \multirow[t]{2}{*}{$p$-value } & \multicolumn{2}{|c|}{ FL at baseline } & \multirow[t]{2}{*}{$p$-value } & \multirow{2}{*}{$\begin{array}{c}\text { Non-FL versus FL } \\
\text { p-value }\end{array}$} \\
\hline & Pre-BD & Post-BD & & Pre-BD & Post-BD & & \\
\hline \multicolumn{8}{|l|}{ FEV 1} \\
\hline $\mathrm{L}$ & $1.27 \pm 0.43$ & $1.47 \pm 0.53$ & $<0.001$ & $0.95 \pm 0.27$ & $1.14 \pm 0.30$ & $<0.001$ & 0.007 \\
\hline$\%$ pred & $47.89 \pm 14.55$ & $54.72 \pm 17.34$ & $<0.001$ & $36.10 \pm 10.07$ & $43.15 \pm 11.89$ & $<0.001$ & 0.004 \\
\hline L & $2.54 \pm 0.70$ & $2.91 \pm 0.77$ & $<0.001$ & $2.28 \pm 0.65$ & $2.80 \pm 0.77$ & $<0.001$ & 0.213 \\
\hline$\%$ pred & $73.22 \pm 14.64$ & $83.26 \pm 13.86$ & $<0.001$ & $63.70 \pm 12.32$ & $78.60 \pm 16.38$ & $<0.001$ & 0.029 \\
\hline \multicolumn{8}{|l|}{ svc } \\
\hline$L$ & $2.61 \pm 0.72$ & $2.90 \pm 0.83$ & $<0.001$ & $2.59 \pm 0.70$ & $2.99 \pm 0.84$ & $<0.001$ & 0.943 \\
\hline$\%$ pred & $74.47 \pm 13.56$ & $82.77 \pm 16.16$ & $<0.001$ & $71.40 \pm 11.68$ & $82.80 \pm 16.94$ & $<0.001$ & 0.439 \\
\hline \multicolumn{8}{|l|}{ IC } \\
\hline$\%$ pred & $216.81 \pm 57.88$ & $203.69 \pm 46.28$ & $<0.001$ & $255.00 \pm 54.92$ & $238.11 \pm 50.72$ & 0.015 & 0.035 \\
\hline \multicolumn{8}{|l|}{ TGV } \\
\hline L & $5.16 \pm 1.33$ & $4.93 \pm 1.24$ & $<0.001$ & $6.14 \pm 1.49$ & $5.84 \pm 1.39$ & 0.019 & 0.030 \\
\hline$\%$ pred & $172.53 \pm 33.05$ & $164.73 \pm 31.44$ & $<0.001$ & $197.65 \pm 35.64$ & $188.28 \pm 33.75$ & 0.019 & 0.023 \\
\hline \multicolumn{8}{|l|}{ TLC } \\
\hline L & $7.02 \pm 1.66$ & $6.97 \pm 1.61$ & 0.261 & $7.99 \pm 1.67$ & $7.97 \pm 1.57$ & 0.747 & 0.070 \\
\hline$\%$ pred & $125.46 \pm 19.76$ & $124.48 \pm 17.33$ & 0.184 & $138.65 \pm 18.38$ & $137.94 \pm 15.73$ & 0.703 & 0.031 \\
\hline \multicolumn{8}{|l|}{ Zrs $5 \mathrm{~Hz}$} \\
\hline $\bar{R}$ insp & $4.4 \pm 1.2$ & $3.8 \pm 1.5$ & 0.014 & $5.8 \pm 1.8$ & $4.6 \pm 1.3$ & $<0.001$ & 0.005 \\
\hline $\bar{R} \exp$ & $5.4 \pm 1.5$ & $4.9 \pm 1.6$ & 0.032 & $7.0 \pm 3.0$ & $6.9 \pm 3.3$ & 0.511 & 0.038 \\
\hline $\bar{R}$ tot & $5.0 \pm 1.4$ & $4.5 \pm 1.5$ & 0.024 & $6.6 \pm 2.5$ & $6.1 \pm 2.6$ & 0.020 & 0.013 \\
\hline
\end{tabular}

Data are presented as mean $\pm \mathrm{SD}$, unless otherwise stated. Impedance data $(R$ and $X)$ are expressed as $\mathrm{cmH}_{2} \mathrm{O} \cdot \mathrm{S} \cdot \mathrm{L}^{-1}$. BD: bronchodilator; FEV 1 : forced expiratory volume in $1 \mathrm{~s}$; \% pred: \% predicted; FVC: forced vital capacity; SVC: slow vital capacity; IC: inspiratory capacity; RV: residual volume; TGV: thoracic gas volume; TLC: total lung capacity; Zrs: within-breath respiratory system input impedance; $\bar{R}$ insp: mean inspiration resistance; $\bar{R}$ exp: mean expiration resistance; $\bar{R}$ tot: mean whole breath resistance; $\bar{X}$ insp: mean reactance during inspiration; $\bar{X}_{\text {exp }}$; mean reactance during expiration: $\bar{X}_{\text {tot: }}$ mean total reactance ; $\Delta \bar{X}_{\text {rs: }}$ : mean difference in reactance; $\bar{R} 5-\bar{R} 19$ : mean difference in resistances at 5 and $19 \mathrm{~Hz} .{ }^{*}$ : the p-values are reported for the paired comparison pre- and post-BD in each group and for the unpaired comparison between non-FL and FL at baseline. All values in bold are statistically significant.

The changes in oscillatory mechanics noted above were largely driven by changes in flow-limited patients, presumably because the fall in lung volume in the nonflow-limited patients compensated for any improvement in resting resistance or reactance. The fall in group mean Rrs in the flow-limited subjects was due mainly to a reduction in $\bar{R}$ insp of the respiratory system.

Multifrequency testing generated large amounts of data, which have been retained for completeness along with the breathing pattern data as online supplementary material. In general, these showed qualitatively similar changes in response to the bronchodilator to the data measured at $5 \mathrm{~Hz}$. We characterised the heterogeneity of the lung with an index of the frequency dependence of resistance, $\bar{R} 5-\bar{R} 19$. It is possible to identify at least four different sources of heterogeneity: serial distribution of airway geometry [37], heterogeneous parallel airway constriction pattern [38], airway wall shunting [30], and heterogeneity of alveolar tissue in heterogeneous parenchymal diseases, such as emphysema [39]. Serial distribution of airway geometry affects impedance data mainly for frequencies $>100 \mathrm{~Hz}$, providing only negligible contribution at the forcing frequencies used in this study [40]. Tissue heterogeneity should not be affected by the administration of BD. As the frequency dependence of Rrs changed statistically significantly after bronchodilator application in most of the patients, with 
some of them showing no frequency dependence at all after $\mathrm{BD}$, the tissue heterogeneity should not be the dominant mechanism affecting $\bar{R} 5-\bar{R} 19$. Parallel airway constriction and airway wall shunting are not easy to differentiate, and it is likely that they are all together contributing to the definition of $\bar{R} 5-\bar{R} 19$.

Between-frequency comparisons showed that nonflow-limited patients had a relatively homogeneous distribution of resistance, and their response to the bronchodilator was similar whether $\bar{R}$ tot or $\bar{R}$ insp was plotted. By contrast, flow-limited patients showed a much greater frequency dependence at baseline, suggesting a highly heterogeneous pattern of obstruction. This pattern was apparently little affected by the bronchodilator when total Rrs was considered. However, a clear fall in frequency dependence of $R$ rs was evident when inspiratory data, unaffected by the artefacts due to EFL, were used. This suggests that BD has a great effect in homogenising time constants throughout the airway tree in flow-limited patients, as also supported by the changes in dynamic elastance with frequency, which are also in agreement with the model prediction of LUTCHEN et al. [26].

In some patients, bronchodilators abolished expiratory flow limitation in all or in the majority of breaths. This has been seen in other reports [31], although the potential for breath-tobreath variation in the presence of flow limitation complicates the interpretation of data when only a few breaths are sampled, as with the negative expiratory pressure method for identifying EFL. This subset of patients did not differ either in their baseline physiological characteristics before testing or in their degree of within-breath reactance change either before or after treatment.

Our data have some limitations. All studies were conducted at rest and seated, and changes in lung mechanics may not translate to data during exercise, although as already noted there is a good relationship between resting operating lung volumes and exercise performance. Oscillatory signals can be influenced by the shunt compliance provided by the upper airway in COPD. However, each subject is their own control in our data before and after the bronchodilator. Changes related to technical factors, such as the presence of expiratory flow limitation, provide a plausible explanation for the limited bronchodilator response previously reported in COPD using total Zrs data and attributed to upper airway factors [15]. Our data have been reported using the multifrequency pseudorandom noise signal, which might have yielded different results to previous single frequency oscillation studies. However, as indicated in the online supplementary material, any differences seen with the systems are likely to relate to physiological differences between breath variation in the degree of flow limitation rather than systematic methodological error. This issue is considered in more detail in the online supplementary material.

In summary, expiratory flow limitation during tidal breathing has an important influence on the changes in resting lung mechanics after bronchodilator drugs in COPD, but it does not predict the magnitude of the subsequent improvement in operating lung volume, at least not at rest. Noninvasive measurements of tidal lung mechanics using the forced oscillation method are an attractive alternative to more usual effort dependent tests of pulmonary function and others have shown that such tests are a sensitive way of detecting bronchodilator effects in these patients [41]. However, the change in the total $\mathrm{Zrs}$ after a bronchodilator may underestimate the true effects of therapy if expiratory impedance data are not excluded from the analysis in flow-limited patients. Despite these limitations, forced-oscillation data add considerable insight into the way treatment works in COPD and, as a noninvasive, effort-independent methodology, is well suited for monitoring patients in clinical settings where reliable clinical measurement has until now been difficult.

\section{REFERENCES}

1 Celli BR, MacNee W. Standards for the diagnosis and treatment of patients with COPD: a summary of the ATS/ ERS position paper. Eur Respir J 2004; 23: 932-946.

2 Dellaca RL, Duffy N, Pompilio PP, et al. Expiratory flow limitation detected by forced oscillation and negative expiratory pressure. Eur Respir J 2007; 29: 363-374.

3 Calverley PM, Koulouris NG. Flow limitation and dynamic hyperinflation: key concepts in modern respiratory physiology. Eur Respir J 2005; 25: 186-199.

4 Diaz O, Villafranca C, Ghezzo $\mathrm{H}$, et al. Breathing pattern and gas exchange at peak exercise in COPD patients with and without tidal flow limitation at rest. Eur Respir J 2001; 17: $1120-1127$.

5 Newton MF, O'Donnell DE, Forkert L. Response of lung volumes to inhaled salbutamol in a large population of patients with severe hyperinflation. Chest 2002; 121: 1042-1050.

6 Calverley PM, Burge PS, Spencer S, et al. Bronchodilator reversibility testing in chronic obstructive pulmonary disease. Thorax 2003; 58: 659-664.

7 Anthonisen NR, Lindgren PG, Tashkin DP, et al. Bronchodilator response in the lung health study over 11 yrs. Eur Respir J 2005; 26: 45-51.

8 O'Donnell DE, Sciurba F, Celli B, et al. Effect of fluticasone propionate/salmeterol on lung hyperinflation and exercise endurance in COPD. Chest 2006; 130: 647-656.

9 O'Donnell DE, Lam M, Webb KA. Spirometric correlates of improvement in exercise performance after anticholinergic therapy in chronic obstructive pulmonary disease. Am J Respir Crit Care Med 1999; 160: 542-549.

10 Tantucci C, Duguet A, Similowski T, et al. Effect of salbutamol on dynamic hyperinflation in chronic obstructive pulmonary disease patients. Eur Respir J 1998; 12: 799-804.

11 Boni E, Corda L, Franchini D, et al. Volume effect and exertional dyspnoea after bronchodilator in patients with COPD with and without expiratory flow limitation at rest. Thorax 2002; 57: 528-532.

12 Koulouris NG, Valta P, Lavoie A, et al. A simple method to detect expiratory flow limitation during spontaneous breathing. Eur Respir J 1995; 8: 306-313.

13 Dellaca RL, Santus P, Aliverti A, et al. Detection of expiratory flow limitation in COPD using the forced oscillation technique. Eur Respir J 2004; 23: 232-240.

14 Dellacà RL, Rotger $\mathrm{M}$, Aliverti $\mathrm{A}$, et al. Noninvasive detection of expiratory flow limitation in COPD patients during nasal CPAP. Eur Respir J 2006; 27: 983-991. 
15 van Noord JA, Smeets J, Clement J, et al. Assessment of reversibility of airflow obstruction. Am J Respir Crit Care Med 1994; 150: 551-554.

16 Farre R, Peslin R, Rotger M, et al. Forced oscillation total respiratory resistance and spontaneous breathing lung resistance in COPD patients. Eur Respir J 1999; 14: 172-178.

17 Chalker RB, Celli BR, Habib RH, et al. Respiratory input impedance from 4 to $256 \mathrm{~Hz}$ in normals and chronic airflow obstruction: comparisons and correlations with spirometry. Am Rev Respir Dis 1992; 146: 570-576.

18 Rabe KF, Beghe B, Luppi F, et al. Update in chronic obstructive pulmonary disease 2006. Am J Respir Crit Care Med 2007; 175: 1222-1232.

19 Miller MR, Hankinson J, Brusasco V, et al. Standardisation of spirometry. Eur Respir J 2005; 26: 319-338.

20 Quanjer PH, Tammeling GJ, Cotes JE, et al. Lung volumes and forced ventilatory flows. Report Working Party Standardization of Lung Function Tests, European Community for Steel and Coal. Official Statement of the European Respiratory Society. Eur Respir J 1993; 6: Suppl. $16,5-40$.

21 Suki B, Lutchen KR. Pseudorandom signals to estimate apparent transfer and coherence functions of nonlinear systems: applications to respiratory mechanics. IEEE Trans Biomed Eng 1992; 39: 1142-1151.

22 Grimby G, Takishima T, Graham W, et al. Frequency dependence of flow resistance in patients with obstructive lung disease. J Clin Invest 1968; 47: 1455-1465.

23 Peslin R, Duvivier C, Didelon J, et al. Respiratory impedance measured with head generator to minimize upper airway shunt. J Appl Physiol 1985; 59: 1790-1795.

24 Kaczka DW, Ingenito EP, Lutchen KR. Technique to determine inspiratory impedance during mechanical ventilation: implications for flow limited patients. Ann Biomed Eng 1999; 27: 340-355.

25 Kaczka DW, Barnas GM, Suki B, et al. Assessment of timedomain analyses for estimation of low-frequency respiratory mechanical properties and impedance spectra. Ann Biomed Eng 1995; 23: 135-151.

26 Lutchen KR, Greenstein JL, Suki B. How inhomogeneities and airway walls affect frequency dependence and separation of airway and tissue properties. J Appl Physiol 1996; 80: 1696-1707.

27 Pellegrino R, Viegi G, Brusasco V, et al. Interpretative strategies for lung function tests. Eur Respir J 2005;26:948-968.
28 Metz CE. Basic principles of ROC analysis. Semin Nucl Med 1978; 8: 283-298.

29 Milic-Emili J. Expiratory flow limitation: Roger S. Mitchell Lecture. Chest 2000; 117: Suppl. 1, 219S-223S.

30 Otis AB, Mc Kerrow CB, Bartlett RA, et al. Mechanical factors in distribution of pulmonary ventilation. J Appl Physiol 1956; 8: 427-443.

31 Hadcroft J, Calverley PM. Alternative methods for assessing bronchodilator reversibility in chronic obstructive pulmonary disease. Thorax 2001; 56: 713-720.

32 Tashkin DP, Celli B, Decramer M, et al. Bronchodilator responsiveness in patients with COPD. Eur Respir J 2008; 31: 742-750.

33 O'Donnell DE, Fluge T, Gerken F, et al. Effects of tiotropium on lung hyperinflation, dyspnoea and exercise tolerance in COPD. Eur Respir J 2004; 23: 832-840.

34 Johnson MK, Birch M, Carter R, et al. Measurement of physiological recovery from exacerbation of chronic obstructive pulmonary disease using within-breath forced oscillometry. Thorax 2007; 62: 299-306.

35 Stevenson NJ, Walker PP, Costello RW, et al. Lung mechanics and dyspnea during exacerbations of chronic obstructive pulmonary disease. Am J Respir Crit Care Med 2005; 172: 1510-1516.

36 O'Donnell DE, Voduc N, Fitzpatrick M, et al. Effect of salmeterol on the ventilatory response to exercise in chronic obstructive pulmonary disease. Eur Respir J 2004; 24: 86-94.

37 Habib RH, Suki B, Bates JH, Jackson AC. Serial distribution of airway mechanical properties in dogs: effects of histamine. J Appl Physiol 1994; 77: 554-566.

38 Gillis HL, Lutchen KR. How heterogeneous bronchoconstriction affects ventilation distribution in human lungs: a morphometric model. Ann Biomed Eng 1999; 27: 14-22.

39 Ito S, Ingenito EP, Arold SP, et al. Tissue heterogeneity in the mouse lung: effects of elastase treatment. J Appl Physiol 2004; 97: 204-212.

40 Jackson AC, Suki B, Ucar M, et al. Branching airway network models for analyzing high-frequency lung input impedance. J Appl Physiol 1993; 75: 217-227.

41 Borrill ZL, Houghton CM, Tal-Singer R, et al. The use of plethysmography and oscillometry to compare long-acting bronchodilators in patients with COPD. Br J Clin Pharmacol 2008; 65: 244-252. 